\title{
A QCD description of the ATLAS jet veto measurement
}

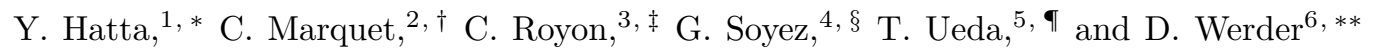 \\ ${ }^{1}$ Faculty of Pure and Applied Sciences, University of Tsukuba, Tsukuba, Ibaraki 305-8571, Japan \\ ${ }^{2}$ Centre de physique théorique, École Polytechnique, CNRS, 91128 Palaiseau, France \\ ${ }^{3}$ IRFU/Service de physique des particules, CEA/Saclay, 91191 Gif-sur-Yvette cedex, France \\ ${ }^{4}$ Institut de physique théorique, CEA/Saclay, 91191 Gif-sur-Yvette cedex, France \\ ${ }^{5}$ Institut für Theoretische Teilchenphysik, Karlsruhe Institute of Technology (KIT) D-76128 Karlsruhe, Germany \\ ${ }^{6}$ Department of Physics and Astronomy, Uppsala University, Box 516, SE-751 20 Uppsala, Sweden
}

We present a new QCD description of the ATLAS jet veto measurement, using the BanfiMarchesini-Smye equation to constrain the inter-jet QCD radiation. This equation resums emissions of soft gluons at large angles, at leading-logarithmic accuracy, and accounts for both the so-called Sudakov and non-global logarithms. We show that this approach is able to reproduce, with no fitting parameters, the fraction of high- $p_{T}$ forward/backward di-jet events which do not contain additional hard emissions in the inter-jet rapidity range. We also compute the gap fraction in fixed-order perturbation theory to $\mathcal{O}\left(\alpha_{s}^{2}\right)$ and show that the perturbative series is unstable at large rapidity intervals.

\section{INTRODUCTION}

Recently the ATLAS collaboration measured, in proton-proton collisions at the LHC, the fraction of di-jet events that do not contain additional hard radiation in the inter-jet rapidity range [1]. The original goal of this measurement was to look for BFKL-type effects, as was previously done at the Tevatron with the so-called 'jet-gap-jet' observable 2 25. However the use of a veto scale $E_{\text {out }} \gg \Lambda_{Q C D}$ by ATLAS, instead of a true rapidity gap void of any hadronic activity, drastically reduces the sensitivity to BFKL physics. Rather, it turns out that this 'jet-veto' measurement is sensitive to the physics of inter-jet energy flow, and in the limit $p_{T} \gg E_{\text {out }}$, to the resummation of soft large-angle gluon emissions in perturbative QCD.

In that regime, the picture that emerges from the comparison [1] between the ATLAS data and Monte-Carlo predictions is far from clear: while HERWIG [6] and PYTHIA [7] tend to be in reasonable agreement with the measurement, fixed-order [8, 9] calculations matched with parton shower are quite below the data. Furthermore, adding BFKL logarithms, as done e.g. in HEJ [10, to take into account the fact that the rapidity difference between the two primary jets, $\Delta y$, can be large does not help. A possible origin of these shortcomings may be the insufficiency of the angular-ordered parton shower to faithfully capture the physics of energy flow. Indeed, the soft gluons which constitute inter-jet radiation are not ordered in angle but rather in $p_{T}$, which makes the relevant resummation single$\operatorname{logarithmic~}\left(\alpha_{s} \ln p_{T} / E_{\text {out }}\right)^{n}$.

The main goal of this paper is to provide a QCD-based computation of the jet veto cross-section obtained by resumming these logarithmically-enhanced $\left(\alpha_{s} \ln p_{T} / E_{\text {out }}\right)^{n}$ term: ${ }^{n}$ There are actually two types of such logarithms the Sudakov logs and the non-global logs. The former is more well-known and can be resummed by using the soft anomalous dimension technique [11, 12]. On the other hand, the non-global logs arise from soft emissions from the secondary gluons (not from the primary hard partons), and can only be resummed in the large- $N_{c}$ limit [13] where the successive emission of soft gluons may be viewed as the splitting of color dipoles? ${ }^{2}$. While HERWIG and PYTHIA can partly account for the effect of these logarithms due to some overlap in the phase space [15], in principle they are not optimized for observables such as the gap fraction.

In this paper we investigate whether the ATLAS data can be described by a perturbative framework which incorporates the relevant single-logarithms to all orders. For the resummation of the non-global logs, we follow the approach of Banfi, Marchesini and Smye (BMS) [16] who reduced the problem to solving a nonlinear integro-differential equation. In a previous paper [17, two of us numerically solved this equation and estimated the survival probability of the BFKL-induced rapidity gap. Here we consider the one-gluon exchange (octet) channel and compute the gap fraction in proton-proton collisions to be directly compared with the ATLAS data. An approach similar in spirit was

\footnotetext{
* hatta@het.ph.tsukuba.ac.jp

$\dagger$ cyrille.marquet@cern.ch

$\ddagger$ christophe.royon@cea.fr

$\S \overline{\text { gregory.soyez@cea.fr }}$

ฯ takahiro.ueda@kit.edu

** ḋominik.werder@ipnysics.uu.se

1 The fact that A'TLAS quotes very small non-perturbative effects (smaller than $2 \%$ ) on their measurement of the gap fraction means that we may hope to achieve a good description of the jet veto cross-sections based purely on perturbative QCD.

2 This process is mathematically identical to the gluon splitting in the BFKL evolution [14.
} 
taken in [12, 18 where the authors did not use the large- $N_{c}$ approximation and fully included the Sudakov logs. The non-global logs, however, were included only by introducing a $K$-factor. For other recent discussions of energy flow and the ATLAS data, see [19-22].

The plan of the paper is as follows. In Section II, we first compute the gap fraction in fixed-order perturbation theory to $\mathcal{O}\left(\alpha_{s}^{2}\right)$ and point out a problem which arises when the rapidity gap $\Delta y$ becomes large. In Section III, we describe the BMS approach which incorporates resummation and compute the gap fraction in this framework. The results are then compared with the ATLAS data. Finally Section IV is devoted to conclusion.

\section{FIXED-ORDER COMPUTATION}

The observable of interest is the gapped fraction in proton-proton collisions measured by the ATLAS collaboration at $\sqrt{s}=7 \mathrm{TeV}$. It is defined by

$$
\mathcal{R}\left(\Delta y, p_{T}\right) \equiv \frac{d \sigma^{\text {veto }}}{d \Delta y d^{2} p_{T}} / \frac{d \sigma^{\text {incl }}}{d \Delta y d^{2} p_{T}},
$$

where $\sigma^{\text {incl }}$ is the inclusive cross section of di-jet events and $\Delta y$ is the rapidity difference of the two jets which have mean transverse momentum $p_{T}=\left(p_{T, 1}+p_{T, 2}\right) / 2 \geq 50 \mathrm{GeV}$ and rapidity $\left|y_{i}\right|<4$.4. Jets are reconstructed using the anti- $k_{t}$ algorithm [23] with the radius parameter $\bar{R}=0.6$. In defining the di-jet system in each event, ATLAS used two different selection criteria: The highest- $p_{T}$ jet pair and the most forward/backward jet pair. $\sigma^{\text {veto }}$ is the gapped cross section in which a veto is applied to the di-jet cross section requiring that no jet with $p_{T}$ above $E_{\text {out }}=20 \mathrm{GeV}$ is observed in the rapidity interval between the two jets.

As explained in the introduction, one expects in the perturbative computation of the ratio (1) large logarithms of the form $\left(\alpha_{s} \ln p_{T} / E_{\text {out }}\right)^{n}$ to become important. In particular, this includes the Sudakov-type logarithms $\left(\alpha_{s} \Delta y \ln p_{T} / E_{\text {out }}\right)^{n}$ which are additionally enhanced by factors of $\Delta y$ when $\Delta y$ is large. A fixed-order computation should then break down and the large logarithms should be resummed in order to obtain a correct description of the vetoed di-jet cross section. However, before coming to that resummation, it remains interesting to check around which value of $\Delta y$ the perturbative series becomes unstable. Therefore, in this section we first perform a fixed-order study of the veto fraction.

Since the leading-order $\left(\mathcal{O}\left(\alpha_{s}^{2}\right)\right)$ vetoed and inclusive cross-sections are equal, the first non-trivial order for $\mathcal{R}$ is when the cross-sections are computed at NLO. At first sight, the fact that the inclusive jet cross-section is not known at NNLO, because of its 2-loop pure-virtual contribution, seems to indicate that the story ends here and $\mathcal{R}$ can only be computed at $\mathcal{O}\left(\alpha_{s}\right)$. But, since this 2-loop pure-virtual contribution to the jet cross-section is actually the same in the vetoed and inclusive cases, one can show that, following the same arguments as in [24, this unknown piece does not contribute to the $\mathcal{O}\left(\alpha_{s}^{2}\right)$ term in the series expansion of $\mathcal{R}$. An explicit series expansion shows that one can write $\mathcal{R}$ as 3

$$
\mathcal{R}\left(\Delta y, p_{T}\right)=1-\frac{\left(\frac{d^{2} \sigma^{\text {noveto }}}{d p_{T} d \Delta y}\right)_{L O}}{\left(\frac{d^{2} \sigma^{\text {incl }}}{d p_{T} d \Delta y}\right)_{L O}} \alpha_{s}+\left[\frac{\left(\frac{d^{2} \sigma^{\text {noveto }}}{d p_{T} d \Delta y}\right)_{L O}\left(\frac{d^{2} \sigma^{\text {incl }}}{d p_{T} d \Delta y}\right)_{N L O}}{\left(\frac{d^{2} \sigma^{\text {incl }}}{d p_{T} d \Delta y}\right)_{L O}^{2}}-\frac{\left(\frac{d^{2} \sigma^{\text {noveto }}}{d p_{T} d \Delta y}\right)_{N L O}}{\left(\frac{d^{2} \sigma^{\text {incl }}}{d p_{T} d \Delta y}\right)_{L O}} \alpha_{s}^{2}\right.
$$

where the $\alpha_{s}$ dependence has been explicitly factored out and $\sigma^{\text {noveto }}=\sigma^{\text {incl }}-\sigma^{\text {veto }}$.

All the differential cross-sections in (2) can be computed explicitly using NLOJet++ 25]. Following the ATLAS analysis [1] as described above and focusing, for definiteness, on the case where the di-jet system is made of the most forward and most backward jets, we simulated events with NLOJet $++\mathrm{v} 4.1 .2$ using the anti- $k_{t}$ algorithm as implemented in FastJet [26. In the perturbative computation we have set both the renormalization and factorization scales ${ }^{4}$ to $p_{T}$ and we have used the MRST2002 PDF set at NLO [27].

The result of this analysis is shown on Fig. 1 where $\mathcal{R}$ is plotted as a function of the rapidity interval $\Delta y$ for different bins in $p_{T}$. The dashed (red) curves are obtained by truncating (2) at order $\alpha_{s}$ while the solid (blue) curves also keep the $\mathcal{O}\left(\alpha_{s}^{2}\right)$ terms. We immediately notice that the LO result becomes negative when $\Delta y$ becomes large while the NLO result becomes larger than 1 . These unphysical results are actually not surprising and stem from the fact that at large rapidity interval $\Delta y$ one expects a Sudakov-type behavior of the form $\mathcal{R} \propto \exp \left(-C \alpha_{s} \Delta y \ln p_{T}\right)$ which,

3 An alternative and maybe more direct approach is to write $\mathcal{R}=1-\sigma^{\text {noveto }} / \sigma^{\text {incl }}$ and to realize that taking both cross-sections at NLO, i.e. $\sigma^{\text {noveto }}$ at $\mathcal{O}\left(\alpha_{s}^{4}\right)$ and $\sigma^{\text {incl }}$ at $\mathcal{O}\left(\alpha_{s}^{3}\right)$, would formally give a description of $\mathcal{R}$ at $\mathcal{O}\left(\alpha_{s}^{2}\right)$, while $\sigma_{N L O}^{\text {noveto }}$ does not depend on the 2 -loop cross-section with only 2 partons in the final-state. The expression in 2 is no more than an explicit series expansion of that statement.

4 Although this would naturally apply for a di-jet system based on the two hardest jets in the event, $p_{T}$ is the natural scale choice also for this forward-backward configuration which is expected to be dominated by a $t$-channel exchange. Note also that we could have studied the scale uncertainty and compared it to the differences between the LO and NLO curves in Fig. 1 


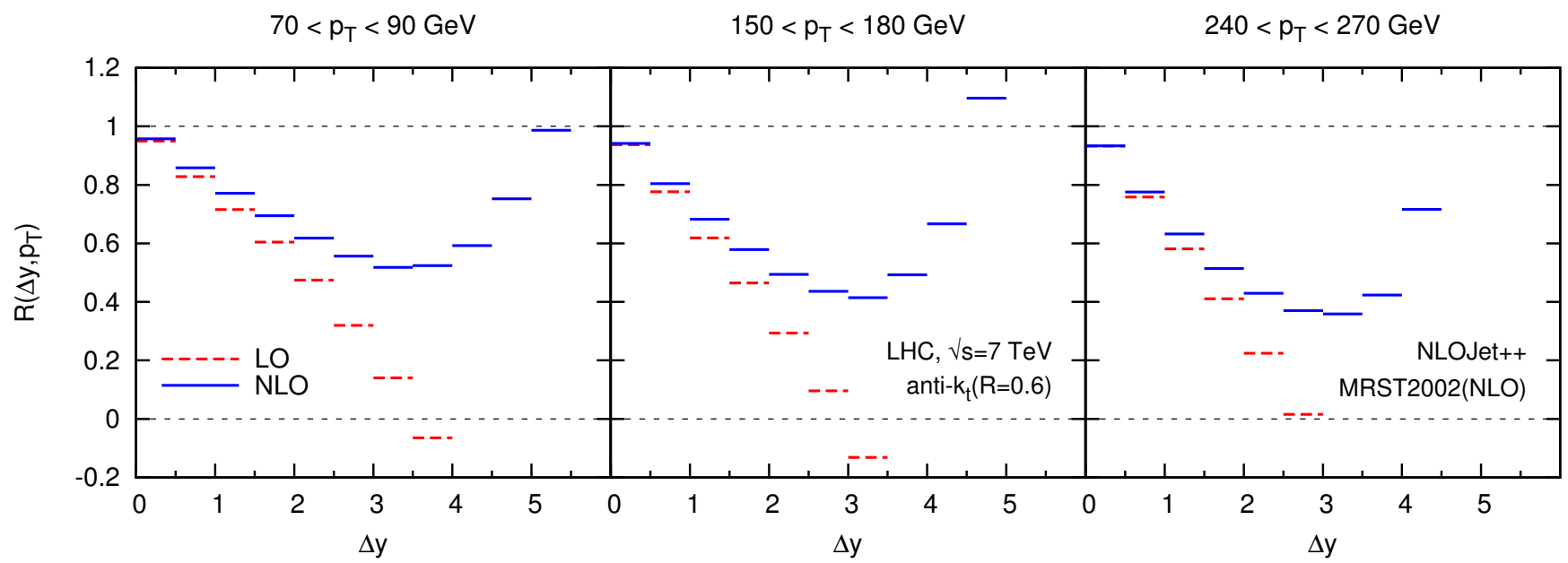

Figure 1. LO $\left(\mathcal{O}\left(\alpha_{s}\right)\right)$ and NLO $\mathcal{O}\left(\alpha_{s}^{2}\right)$ predictions for the fraction of di-jet events with a jet veto.

truncated at fixed order, would give $\mathcal{R} \propto 1-C \alpha_{s} \Delta y \ln p_{T}+\left(C \alpha_{s} \Delta y \ln p_{T}\right)^{2} / 2+\cdots$. More interestingly, one sees explicitly from Fig. 1 that the perturbative series becomes completely unstable for $\Delta y \gtrsim 2$, with a weak dependence on $p_{T}$. The main message of this study is that one should thus expect some form of resummation to be necessary for $\Delta y \gtrsim 2$ which is practically the whole interesting region of the physics of rapidity gap.

\section{RESUMMED COMPUTATION}

In this section we undertake the resummed computation of the gapped fraction $\mathcal{R}$ which should cure the problem with the fixed-order computation we have just seen. Our calculation is based on the approach by Banfi-MarchesiniSmye (BMS). We first describe their approach and adapt it for the problem at hand, and then present the numerical results.

\section{A. The BMS equation}

Consider a pair of cones pointing back-to-back in the direction of the beam axis ( $z$-axis) as in Fig. 2 . The opening angles of the right cone $\theta_{R}$ and the left cone $\theta_{L}$ need not be the same. A quark and an antiquark in the color singlet state ('dipole') are contained in the cones and moving in the directions $\Omega=(\theta, \phi)$ and $\Omega^{\prime}=\left(\theta^{\prime}, \phi^{\prime}\right)$, respectively, with the transverse momentum $p_{T}$. Let $P_{\tau}\left(\theta_{R}, \theta_{L}, \Omega, \Omega^{\prime}\right)$ be the probability that the total amount of energy, or the transverse momentum, emitted from the dipole into the region outside the cones is less than $E_{\text {out }}$. The 'evolution parameter' $\tau$ is related to $E_{\text {out }}$. Taking into account the running of the coupling, we hav $£^{5}[13,28]$ :

$$
\tau=\int_{E_{\text {out }}}^{p_{T}} \frac{d k_{T}}{k_{T}} \frac{\alpha_{s}\left(k_{T}\right) N_{c}}{\pi}=\frac{1}{2 b} \ln \left(\frac{\alpha_{s}\left(E_{\text {out }}\right)}{\alpha_{s}\left(p_{T}\right)}\right),
$$

where $\alpha_{s}\left(p_{T}\right)=\pi /\left(2 b N_{c} \ln \left(p_{T} / \Lambda_{Q C D}\right)\right)$, and $b=\left(11 N_{c}-2 n_{f}\right) / 12 N_{c}$ with $n_{f}=5$ being the number of active flavors.

To zeroth order, $P=1$. Within the large- $N_{c}$ approximation, BMS derived a nonlinear integro-differential equation in $\tau$ [16] which resums all the single logarithms (both Sudakov and non-global) $\left(\alpha_{s} \ln p_{T} / E_{\text {out }}\right)^{n} \sim \tau^{n}$ which appear in the weak coupling expansion of $P$. The numerical solution to this equation has been previously obtained in [16, 17]. Here we solve it again for the kinematic range relevant to the ATLAS data. In doing so, we switch to the rapidity variable

$$
y=\ln \cot \frac{\theta}{2}
$$

\footnotetext{
5 With a fixed coupling approximation, we find $\tau=\left(\alpha_{s} N_{c} / \pi\right) \ln \left(p_{T} / E_{\text {out }}\right)$.
} 


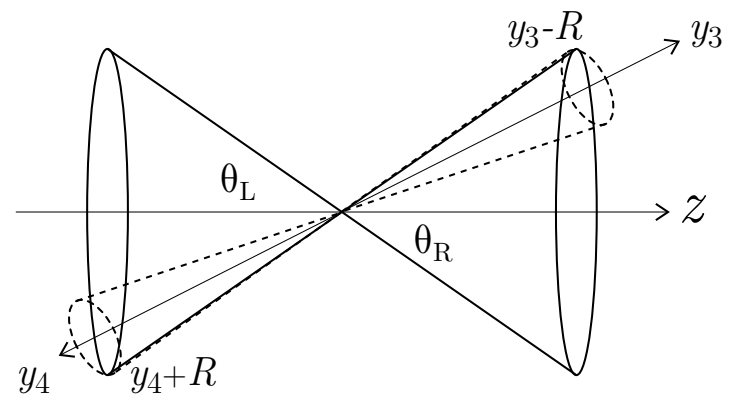

Figure 2. $\theta_{R / L}\left(y_{R / L}=\ln \cot \theta_{R / L} / 2\right)$ is the opening angle (rapidity) of the right/left cone.

instead of the polar angle $\theta$ in the arguments of $P$. Also, we suppress the dependence on the azimuthal angle $\phi$ because $P$ is a function of the difference $\Delta \phi=\phi-\phi^{\prime}$ and below we need solutions only at $\Delta \phi=\pi$. Since the probability $P$ must not depend on a specific Lorentz frame, we can evaluate it in the di-jet c.m.s.

$$
P_{\tau}\left(y_{R}, y_{L}, y, y^{\prime}\right)=P_{\tau}\left(\frac{y_{R}-y_{L}}{2},-\frac{y_{R}-y_{L}}{2}, y-\frac{y_{R}+y_{L}}{2}, y^{\prime}-\frac{y_{R}+y_{L}}{2}\right) .
$$

Eq. (5) allows us to restrict ourselves to the symmetric configuration of the cones, which will greatly facilitate the computations below.

\section{B. The jet-veto cross section in hadron-hadron collisions}

As already suggested by BMS, one can utilize the above probability $P_{\tau}$ to constrain the inter-jet radiation in hadronhadron collisions. The initial and final state soft radiations from a given partonic subprocesses may be deconstructed, in the large- $N_{c}$ approximation, into those from elementary dipoles by inspecting the flow of color. Consider the simplest $p_{1} p_{2} \rightarrow p_{3} p_{4}$ process: $q q^{\prime} \rightarrow q q^{\prime}$ or $\bar{q} \bar{q}^{\prime} \rightarrow \bar{q} \bar{q}^{\prime}$ (different quark flavors). The partonic cross section is given by

$$
\frac{d \sigma_{q q^{\prime}}}{d \hat{t}}=\frac{1}{16 \pi \hat{s}^{2}} h^{A}(\hat{s}, \hat{t}, \hat{u})
$$

where $\hat{s}, \hat{t}$, and $\hat{u}$ are the standard partonic Mandelstam variables $\left(\hat{s}=x_{1} x_{2} s\right.$, etc) and $\left(C_{F}=\left(N_{c}^{2}-1\right) / 2 N_{c}=\frac{4}{3}\right)$

$$
h^{A}(s, t, u)=g^{4} \frac{C_{F}}{N_{c}}\left(\frac{s^{2}+u^{2}}{t^{2}}\right) .
$$

At large- $N_{c}$, and in the one-gluon exchange, color flows as $1 \rightarrow 4$ and $2 \rightarrow 3$. Thus the radiation from the four-parton system (1234) factorizes into that from two dipoles (14) and (23). We require that the amount of energy emitted in the central region bounded by the edges of the jets $p_{3}$ and $p_{4}$ with jet radius $R$ is less than $E_{\text {out }}$ (see, Fig. 2). The cross section with this requirement is given by [16]

$$
\begin{array}{r}
\frac{d \sigma_{q q^{\prime}}^{\text {veto }}}{d \hat{t}}=\frac{1}{16 \pi \hat{s}^{2}}\left(h^{A}(\hat{s}, \hat{t}, \hat{u}) P_{\tau}\left(y_{3}-R, y_{4}+R, \infty, y_{4}\right) P_{\tau}\left(y_{3}-R, y_{4}+R, y_{3},-\infty\right)\right. \\
\left.\quad+h^{A}(\hat{s}, \hat{u}, \hat{t}) P_{\tau}\left(y_{3}-R, y_{4}+R, \infty, y_{3}\right) P_{\tau}\left(y_{3}-R, y_{4}+R, y_{4},-\infty\right)\right) .
\end{array}
$$

Note that we have added the ' $u$-channel' term although there is no $u$-channel diagram for the process $q q^{\prime} \rightarrow q q^{\prime}$, because, when calculating the gapped cross section, we must sum over the two cases $\Delta y=y_{3}-y_{4}>0$ and $\Delta y<0$ since $q$ and $q^{\prime}$ are not identical particles. Adding these two contributions effectively amounts to adding the $u$-channel term but restricting to the $\Delta y>0$ case, facilitating later computation.

By boost invariance (5), we have the relations

$$
\begin{aligned}
& P_{\tau}\left(y_{3}-R, y_{4}+R, \infty, y_{4}\right)=P_{\tau}\left(\frac{\Delta y}{2}-R,-\frac{\Delta y}{2}+R, \infty,-\frac{\Delta y}{2}\right) \equiv P_{14}(\tau, \Delta y), \\
& P_{\tau}\left(y_{3}-R, y_{4}+R, y_{3},-\infty\right)=P_{\tau}\left(\frac{\Delta y}{2}-R,-\frac{\Delta y}{2}+R, \frac{\Delta y}{2},-\infty\right) \equiv P_{23}(\tau, \Delta y),
\end{aligned}
$$




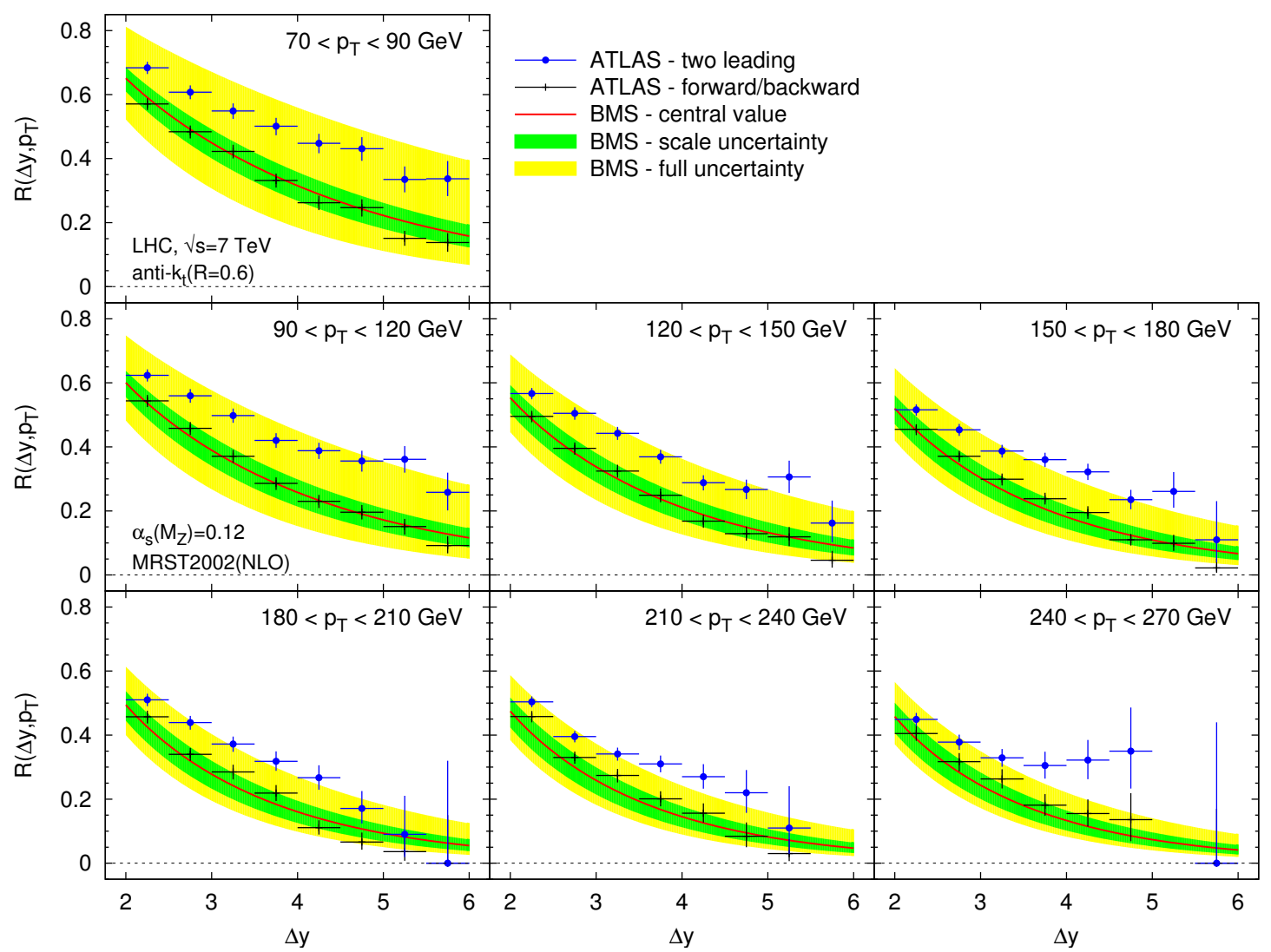

Figure 3. Comparison of the resummed veto fraction with the ATLAS measurement, for a fixed veto energy of $E_{\text {out }}=20 \mathrm{GeV}$, in different bins of $p_{T}$. The inner (green) uncertainty band is obtained taking into account only the renormalization and factorization scale uncertainties, while the outer (yellow) band also includes the subleading logarithmic uncertainty. For the ATLAS data, circles represent the case where the two leading jets are selected while the one where the most forward and backward jets are selected are represented by crosses.

$$
\begin{aligned}
& P_{\tau}\left(y_{3}-R, y_{4}+R, \infty, y_{3}\right)=P_{\tau}\left(\frac{\Delta y}{2}-R,-\frac{\Delta y}{2}+R, \infty, \frac{\Delta y}{2}\right) \equiv P_{13}(\tau, \Delta y), \\
& P_{\tau}\left(y_{3}-R, y_{4}+R, y_{4},-\infty\right)=P_{\tau}\left(\frac{\Delta y}{2}-R,-\frac{\Delta y}{2}+R,-\frac{\Delta y}{2},-\infty\right) \equiv P_{24}(\tau, \Delta y) .
\end{aligned}
$$

Clearly, $P_{14}=P_{23}$ and $P_{13}=P_{24}$, so that (8) takes the simpler form

$$
\begin{aligned}
\frac{d \sigma_{q q^{\prime}}^{\text {veto }}}{d \hat{t}} & =\frac{1}{16 \pi \hat{s}^{2}}\left(h^{A}(\hat{s}, \hat{t}, \hat{u}) P_{14} P_{23}+h^{A}(\hat{s}, \hat{u}, \hat{t}) P_{13} P_{24}\right) \\
& =\frac{1}{16 \pi \hat{s}^{2}}\left(h^{A}(\hat{s}, \hat{t}, \hat{u}) P_{14}^{2}+h^{A}(\hat{s}, \hat{u}, \hat{t}) P_{13}^{2}\right) .
\end{aligned}
$$

In Appendix we list the gapped cross section of all the other partonic subprocesses obtained in a similar way. The results are convoluted with the PDFs to give the hadronic gapped cross section

$$
\frac{d \sigma^{\text {veto }}}{d \Delta y d^{2} p_{T}}=\sum_{i j}^{q, \bar{q}, g} \int_{Y_{\min }}^{Y_{\max }} d Y x_{1} f_{i}\left(x_{1}, p_{T}\right) x_{2} f_{j}\left(x_{2}, p_{T}\right) \frac{1}{\pi} \frac{d \sigma_{i j}^{\text {veto }}}{d \hat{t}}
$$

where $Y=\frac{y_{3}+y_{4}}{2}$ and $Y_{\max }=-Y_{\min }=4.4$ for the ATLAS setup.

\section{Results}

We compute the resummed gapped fraction $\mathcal{R}$ by dividing $(12)$ by the leading-order cross section (that is, the same expression except that $P$ 's are set to 1 everywhere). We have kept $n_{f}=5$ as adequate in the kinematic range under 


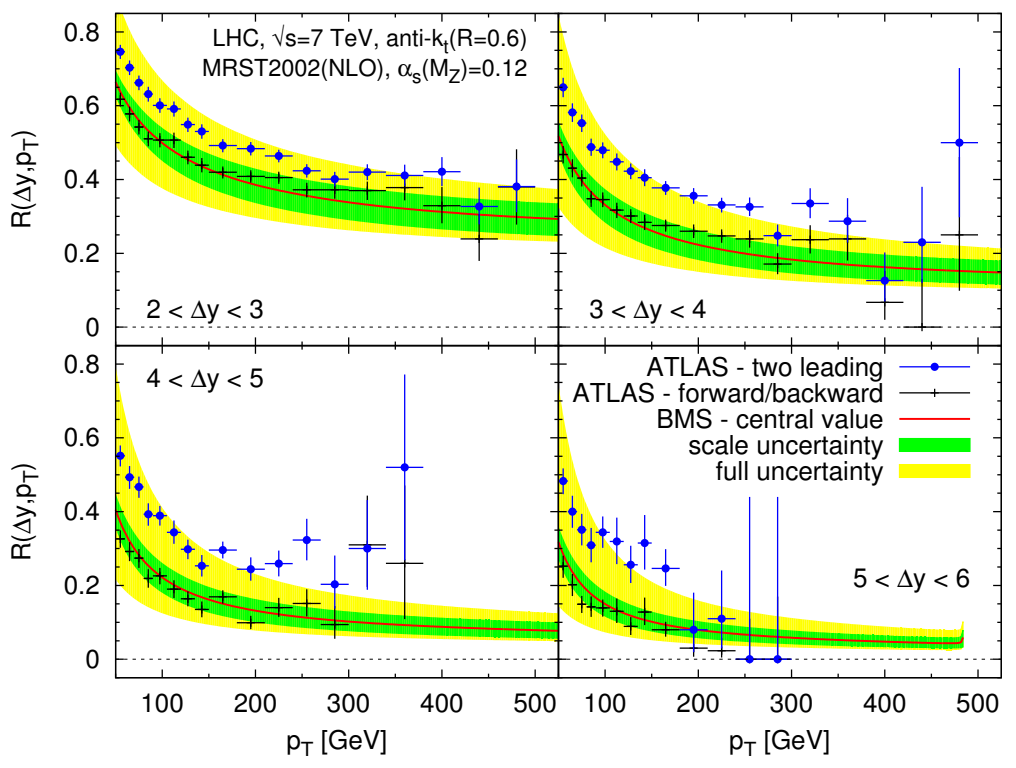

Figure 4. Comparison of the resummed veto fraction with the ATLAS measurement, for a fixed veto energy of $E_{\text {out }}=20 \mathrm{GeV}$, in different bins of $p_{T}$. See Fig. 3 for details.

consideration and fixed the running of the coupling by imposing $\alpha_{s}\left(M_{Z}\right)=0.12$. The theoretical uncertainty on our predictions comes from different sources: First, the renormalization and factorization scale uncertainties are obtained by varying the scale of $\alpha_{s}$ in the matrix elements and in the definition of $\tau$ (Eq. (3) - in the latter case, we vary directly the scale of $\alpha_{s}\left(k_{T}\right)$ in the integrand - as well as the scale $p_{T}$ in the PDFs by a factor of 2 up and down. We exclude the cases where the renormalization scale $\mu_{r}$ and the factorization scale $\mu_{f}$ are $\left(\mu_{r}, \mu_{f}\right)=\left(p_{T} / 2,2 p_{T}\right)$ or $\left(2 p_{T}, p_{T} / 2\right)$. It is interesting to note that the factor $\alpha_{s}^{2}\left(\mu_{r}\right)$ appearing in the matrix elements cancels when taking the cross-section ratio and thus the only renormalization scale uncertainty that remains is the one in the definition of $\tau$. Then, the uncertainty related to subleading logarithmic corrections that are not resummed by the leading $\alpha_{s}^{n} \ln ^{n}\left(p_{T} / E_{\text {out }}\right)$ series is estimated by varying the upper integration bound in the definition of $\tau$, Eq. (3) by a factor of 2 up and down. Finally, the uncertainty band is then taken as the envelope of all the resulting curves.

The results are compared against the ATLAS measurements as published in [1. Since we use the LO cross section where there are only two jets in the final state, we cannot distinguish the two ATLAS data sets based on different di-jet selection criteria. While naively we expect our predictions to be in better agreement with the "two-leading jets" selection, we shall see below that we better reproduce the data based on the most forward/backward jets.

Fig. 3 shows the dependence on the jet rapidity separation for different bins in jet $p_{T}$. We note that the model is overall in good agreement with the measurement. Needless to say, the unphysical behavior, $\mathcal{R}>1$ or $\mathcal{R}<0$, of the fixed-order computation (see Fig. 1) has disappeared. In Fig. (4) the result is presented over the jet $p_{T}$ for different bins in $\Delta y$. In both cases, the agreement with the ATLAS data is good, especially with the sample obtained by selecting the most forward/backward jets. The predictions systematically undershoot the data based on the two leading jets although they remain in agreement once the uncertainty due to subleading effects are taking into account.

Then, Fig. 5 demonstrates the dependence on the jet veto threshold energy $E_{\text {out }}$. We note first that in our results the veto fraction saturates to unity as the threshold $E_{\text {out }}$ approaches the jet $p_{T}$ scale, as expected. In the data, it falls short of unity because of the NLO $(2 \rightarrow 3)$ corrections. On the other hand, the agreement is best for the smaller values of $E_{\text {out }}$, which is expected as well since the formalism we use requires a large scale separation between the jet $p_{T}$ and the threshold $E_{\text {out }}$.

Finally, it is worth mentioning that uncertainties in the choice of the PDF set mostly cancel in the ratio (1). We checked this by trying two different sets, MRST2002 [27] and CT10 [29], and found no noticeable difference.

\section{CONCLUSIONS AND OUTLOOK}

In this paper, we have investigated the QCD resummation of the Sudakov and the non-global logarithms induced by soft gluon emissions in the context of the jet veto cross-section. We conclude that the ATLAS measurement is well described by tree-level QCD supplemented with the jet veto probability calculated perturbatively using the BMS equation which resums both logarithms mentioned above. Actually, the impact of the non-global logarithms is 


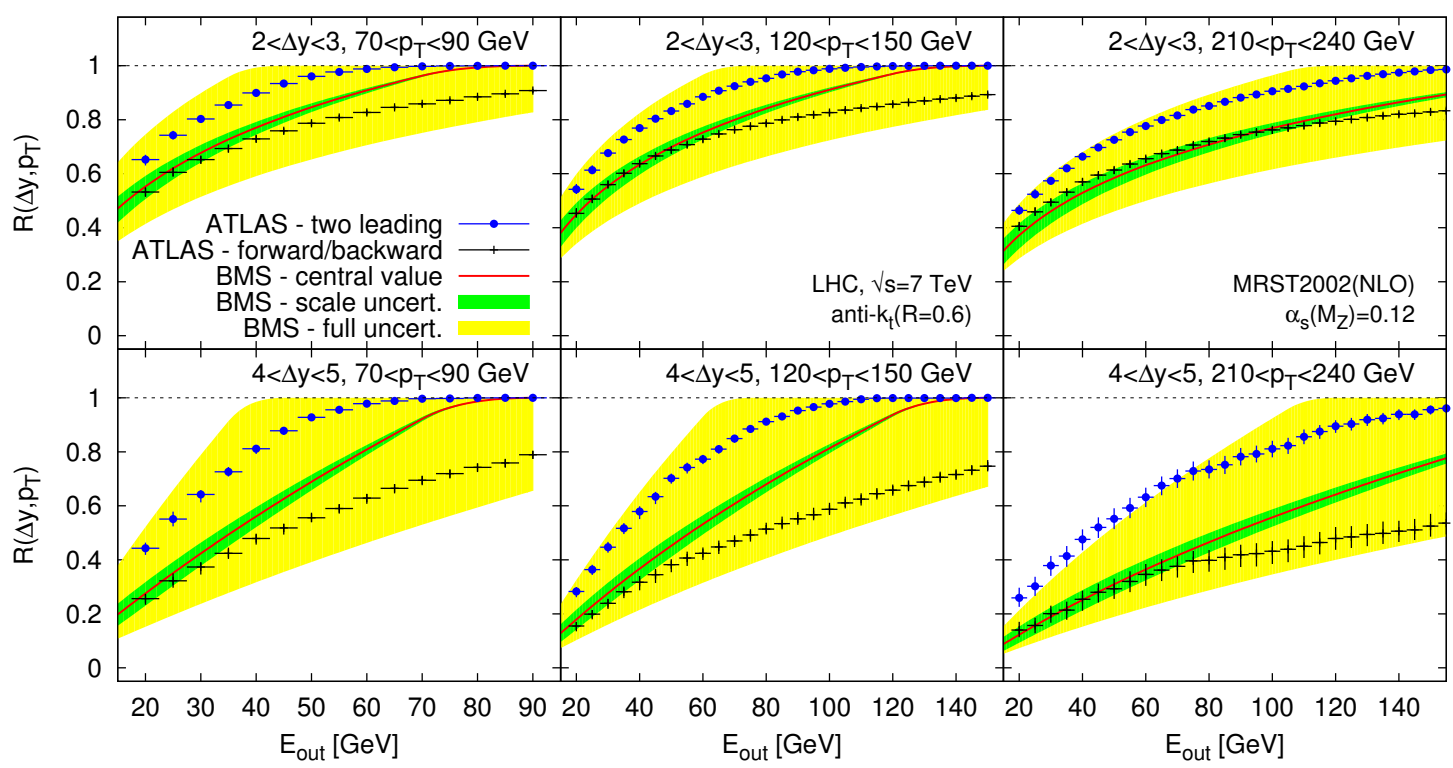

Figure 5. Comparison of the resummed veto fraction with the ATLAS measurement, for different kinematic bins, as a function of the veto threshold $E_{\text {out }}$. See Fig. 3 for details.

modest compared to that of the Sudakov logarithms: with $E_{\text {out }}$ as large as $20 \mathrm{GeV}$, we estimate that the non-global contribution reduces the gap fraction by about $15 \%$.

Having said this, we must comment on uncertainties in our results other than the scale uncertainties already examined. Above all, our description does not allow to disentangle between the two methods proposed by ATLAS to identify the di-jet system. While our approach better reproduces the data where the most forward and the most backward jets are selected, it is quite below the measurement obtained by selecting the two hardest jets in the event. This somewhat goes against the naive expectation that our description should better reproduce the latter situation. This probably means that effects other than the ones included in the BMS equation are at play. Still, we have shown that the resummation of the soft gluon emissions gives a reasonable description of the jet veto probability.

Various additional effects would play a role if we wanted to improve our predictions. First, our approach resums the soft gluon emissions at the leading logarithmic accuracy, so subleading effects would potentially be important. [A rough estimate of this is shown by the yellow band in the figures.] Then, we do not include $1 / N_{c}$ corrections neither in the Sudakov logarithms nor in the non-global ones. The $1 / N_{c}$ corrections in the Sudakov logarithms are fully taken into account in [18, but those for the non-global ones pose a serious theoretical challenge. Also, the NLO $(2 \rightarrow 3)$ corrections to the hard parton cross sections can be important, especially at small $\Delta y$ and for discriminating the two ATLAS data sets corresponding to two different definitions of the di-jet system. In principle, our resummed approach can be extended to $2 \rightarrow 3$ processes, but the flow of color will be considerably more complicated and it is not clear to us at the moment if such an extension is practical. Alternatively, it may be more practical to match the BMS predictions to the fixed-order predictions from Section II which are expected to provide more accurate NLO corrections at least in the small $\Delta y$ region. Finally, we have seen that the data are well described by the color octet contribution alone, without introducing additional BFKL-like (singlet) contributions. As already mentioned in the introduction, the ATLAS choice $E_{\text {out }} \gg \Lambda_{Q C D}$ significantly reduces the sensitivity to BFKL exchanges. Presumably, we need to go to lower values of $E_{\text {out }}$ [17, thus approaching the case of a perfect gap $\left(E_{\text {out }} \rightarrow 0\right)$ [4, 5, 30, to enter the BFKL-dominated regime. It is nevertheless interesting to mention that these corrections would tend to increase our results for the gap fraction $\mathcal{R}$, especially at large $\Delta y$ : Our estimates for $\mathcal{R}$ based on a pure-BFKL calculation are 
in the range 0.8-0.9, and the HEJ event generator, which includes BFKL effects, also predicts values larger than the ones obtained by ATLAS. A combination of the BMS and BFKL contributions could thus bring our predictions in better agreement with the ATLAS measurement obtained based on the two hardest jets in the event. We leave this for future study.

\section{ACKNOWLEDGMENTS}

We thank G. Ingelman, R. Peschanski and G. Salam for discussions and comments. The work of T.U. was supported by the DFG through SFB/TR 9 "Computational Particle Physics". The calculations were partially performed on the HP XC3000 at Steinbuch Centre for Computing of KIT. This work was partially supported by the French Agence Nationale de la Recherche, under the grant ANR-10-CEXC-009-01.

\section{Appendix A: Parton subprocess}

In this Appendix we list the gapped cross section of all the partonic subprocesses which contribute to the sum in (12). Some of them were already considered in [16]. The others are simply obtained by crossing symmetry. We shall need the following building blocks of the cross section [16, 31]

$$
\begin{aligned}
& h^{B}(s, t, u)=g^{4} \frac{C_{F}}{N_{c}}\left(\frac{s^{2}+u^{2}}{t^{2}}+\frac{2}{N_{c}} \frac{s}{t}\right), \\
& h^{C}(s, t, u)=g^{4} C_{F} \frac{u}{t}\left(\frac{t^{2}+u^{2}}{s^{2}}-\frac{1}{N_{c}^{2}}\right), \\
& h^{D}(s, t, u)=2 g^{4} \frac{N_{c}^{2}}{N_{c}^{2}-1}\left(1-\frac{t u}{s^{2}}-\frac{s u}{t^{2}}+\frac{u^{2}}{s t}\right),
\end{aligned}
$$

and the solutions to the BMS equation for different dipole configurations

$$
\begin{aligned}
& P_{\tau}\left(y_{3}-R, y_{4}+R, y_{3}, y_{4}\right)=P_{\tau}\left(\frac{\Delta y}{2}-R,-\frac{\Delta y}{2}+R, \frac{\Delta y}{2},-\frac{\Delta y}{2}\right) \equiv P_{34}(\tau, \Delta y), \\
& P_{\tau}\left(y_{3}-R, y_{4}+R, \infty,-\infty\right)=P_{\tau}\left(\frac{\Delta y}{2}-R,-\frac{\Delta y}{2}+R, \infty,-\infty\right) \equiv P_{12}(\tau, \Delta y) .
\end{aligned}
$$

Note that $P_{34}$ is evaluated at $\Delta \phi=\phi_{3}-\phi_{4}=\pi$ as appropriate for back-to-back jets.

- $q \bar{q}^{\prime} \rightarrow q \bar{q}^{\prime}$ (different quark flavors)

This can be obtained from $q q^{\prime} \rightarrow q q^{\prime}$ by crossing $s \leftrightarrow u$ and $2 \leftrightarrow 4$

$$
\frac{d \sigma_{q \bar{q}^{\prime}}^{\text {veto }}}{d \hat{t}}=\frac{1}{16 \pi \hat{s}^{2}}\left(h^{A}(\hat{u}, \hat{t}, \hat{s}) P_{12} P_{34}+h^{A}(\hat{t}, \hat{u}, \hat{s}) P_{12} P_{34}\right),
$$

where we added a $u$-channel diagram (by the same reason as in (8) which is obtained by crossing $t \leftrightarrow u, 3 \leftrightarrow 4$.

- $q \bar{q} \rightarrow q^{\prime} \bar{q}^{\prime}$ (different quark flavors)

Obtained from $q q^{\prime} \rightarrow q q^{\prime}$ via crossing $s \leftrightarrow t, 2 \leftrightarrow 3$

$$
\begin{aligned}
\frac{d \sigma_{q \bar{q} \rightarrow q^{\prime} \bar{q}^{\prime}}^{\text {veto }}}{d \hat{t}} & =\frac{1}{16 \pi \hat{s}^{2}}\left(h^{A}(\hat{t}, \hat{s}, \hat{u}) P_{14} P_{23}+h^{A}(\hat{u}, \hat{s}, \hat{t}) P_{13} P_{24}\right) \\
& =\frac{1}{16 \pi \hat{s}^{2}}\left(h^{A}(\hat{t}, \hat{s}, \hat{u}) P_{14}^{2}+h^{A}(\hat{u}, \hat{s}, \hat{t}) P_{13}^{2}\right) .
\end{aligned}
$$

- $q q \rightarrow q q, \bar{q} \bar{q} \rightarrow \bar{q} \bar{q}$ (the same flavor) [16]:

$$
\begin{aligned}
\frac{d \sigma_{q q}^{\text {veto }}}{d \hat{t}} & =\frac{1}{16 \pi \hat{s}^{2}}\left(h^{B}(\hat{s}, \hat{t}, \hat{u}) P_{14} P_{23}+h^{B}(\hat{s}, \hat{u}, \hat{t}) P_{13} P_{24}\right) \\
& =\frac{1}{16 \pi \hat{s}^{2}}\left(h^{B}(\hat{s}, \hat{t}, \hat{u}) P_{14}^{2}+h^{B}(\hat{s}, \hat{u}, \hat{t}) P_{13}^{2}\right)
\end{aligned}
$$


Compare with (11). Since the final state particles are identical, one integrates over only half of the phase space, that is, only the $\Delta y>0$ case. Note that the interference term between the two diagrams for $q q \rightarrow q q$ is subleading in $N_{c}$ and does not correspond to a process with definite color flow. It was distributed symmetrically into the second term of $h^{B}$ (A1) [31]. Similar comments apply to the other subleading contributions in $h^{C}$ and $h^{D}$ below.

- $q \bar{q} \rightarrow q \bar{q}$ :

Obtained from $q q \rightarrow q q$ by crossing $s \leftrightarrow u, 2 \leftrightarrow 4$ and adding the $\Delta y<0$ contribution $(t \leftrightarrow u, 3 \leftrightarrow 4)$ because of nonidentical particles in the final state

$$
\begin{aligned}
\frac{d \sigma_{q \bar{q} \rightarrow q \bar{q}}^{\mathrm{veto}}}{d \hat{t}} & =\frac{1}{16 \pi \hat{s}^{2}}\left(h^{B}(\hat{u}, \hat{t}, \hat{s}) P_{12} P_{34}+h^{B}(\hat{u}, \hat{s}, \hat{t}) P_{13} P_{24}+h^{B}(\hat{t}, \hat{u}, \hat{s}) P_{12} P_{34}+h^{B}(\hat{t}, \hat{s}, \hat{u}) P_{14} P_{23}\right) \\
& =\frac{1}{16 \pi \hat{s}^{2}}\left(h^{B}(\hat{u}, \hat{t}, \hat{s}) P_{12} P_{34}+h^{B}(\hat{u}, \hat{s}, \hat{t}) P_{13}^{2}+h^{B}(\hat{t}, \hat{u}, \hat{s}) P_{12} P_{34}+h^{B}(\hat{t}, \hat{s}, \hat{u}) P_{14}^{2}\right) .
\end{aligned}
$$

- $q \bar{q} \rightarrow g g[16]$

$$
\begin{aligned}
\frac{d \sigma_{q \bar{q}}^{\text {veto }}}{d \hat{t}} & =\frac{1}{16 \pi \hat{s}^{2}}\left(h^{C}(\hat{s}, \hat{t}, \hat{u}) P_{34} P_{13} P_{24}+h^{C}(\hat{s}, \hat{u}, \hat{t}) P_{34} P_{14} P_{23}\right) \\
& =\frac{1}{16 \pi \hat{s}^{2}}\left(h^{C}(\hat{s}, \hat{t}, \hat{u}) P_{34} P_{13}^{2}+h^{C}(\hat{s}, \hat{u}, \hat{t}) P_{34} P_{14}^{2}\right) .
\end{aligned}
$$

- $g g \rightarrow q \bar{q}:$

The same as $q \bar{q} \rightarrow g g$ except for the color factor $(3 / 8)^{2}$ and the addition of the $\Delta y<0$ contribution $(t \leftrightarrow u$, $3 \leftrightarrow 4)$

$$
\frac{d \sigma_{g g \rightarrow q \bar{q}}^{\mathrm{veto}}}{d \hat{t}}=\frac{1}{16 \pi \hat{s}^{2}}\left(\frac{3}{8}\right)^{2}\left(h^{C}(\hat{s}, \hat{t}, \hat{u}) P_{34} P_{13}^{2}+h^{C}(\hat{s}, \hat{u}, \hat{t}) P_{34} P_{14}^{2}\right) \times 2
$$

- $q g \rightarrow q g$ and $\bar{q} g \rightarrow \bar{q} g$ :

Obtained from $q \bar{q} \rightarrow g g$ by crossing $s \leftrightarrow t, 2 \leftrightarrow 3$, multiplying the color factor $3 / 8$, adding the $\Delta y<0$ contribution $(t \leftrightarrow u, 3 \leftrightarrow 4)$

$$
\begin{aligned}
\frac{d \sigma_{q g}^{\mathrm{veto}}}{d \hat{t}} & =\frac{-1}{16 \pi \hat{s}^{2}} \frac{3}{8}\left(h^{C}(\hat{t}, \hat{s}, \hat{u}) P_{24} P_{12} P_{34}+h^{C}(\hat{t}, \hat{u}, \hat{s}) P_{24} P_{14} P_{23}+h^{C}(\hat{u}, \hat{s}, \hat{t}) P_{23} P_{12} P_{34}+h^{C}(\hat{u}, \hat{t}, \hat{s}) P_{23} P_{13} P_{24}\right) \\
& =\frac{-1}{16 \pi \hat{s}^{2}} \frac{3}{8}\left(h^{C}(\hat{t}, \hat{s}, \hat{u}) P_{13} P_{12} P_{34}+h^{C}(\hat{t}, \hat{u}, \hat{s}) P_{13} P_{14}^{2}+h^{C}(\hat{u}, \hat{s}, \hat{t}) P_{14} P_{12} P_{34}+h^{C}(\hat{u}, \hat{t}, \hat{s}) P_{14} P_{13}^{2}\right) .(\mathrm{A} 12)
\end{aligned}
$$

Note the overall minus sign.

- $g g \rightarrow g g$ [16]:

$$
\begin{aligned}
\frac{d \sigma_{g g \rightarrow g g}^{\mathrm{veto}}}{d \hat{t}} & =\frac{1}{16 \pi \hat{s}^{2}}\left(h^{D}(\hat{s}, \hat{t}, \hat{u}) P_{12} P_{13} P_{24} P_{34}+h^{D}(\hat{s}, \hat{u}, \hat{t}) P_{12} P_{14} P_{23} P_{34}+h^{D}(\hat{u}, \hat{t}, \hat{s}) P_{14} P_{24} P_{13} P_{23}\right) \\
& =\frac{1}{16 \pi \hat{s}^{2}}\left(h^{D}(\hat{s}, \hat{t}, \hat{u}) P_{12} P_{13}^{2} P_{34}+h^{D}(\hat{s}, \hat{u}, \hat{t}) P_{12} P_{14}^{2} P_{34}+h^{D}(\hat{u}, \hat{t}, \hat{s}) P_{14}^{2} P_{13}^{2}\right) .
\end{aligned}
$$

[1] G. Aad et al. [ATLAS Collaboration], JHEP 1109, 053 (2011).

[2] B. Abbott et al. [D0 Collaboration], Phys. Lett. B 440, 189 (1998).

[3] F. Abe et al. [CDF Collaboration], Phys. Rev. Lett. 80, 1156 (1998).

[4] F. Chevallier, O. Kepka, C. Marquet and C. Royon, Phys. Rev. D 79, 094019 (2009).

[5] O. Kepka, C. Marquet and C. Royon, Phys. Rev. D 83, 034036 (2011). 
[6] M. Bahr, S. Gieseke, M. A. Gigg, D. Grellscheid, K. Hamilton, O. Latunde-Dada, S. Platzer and P. Richardson et al., Eur. Phys. J. C 58 (2008) 639.

[7] T. Sjostrand, S. Mrenna and P. Skands, JHEP 0605 (2006) 026.

[8] M. L. Mangano, M. Moretti, F. Piccinini, R. Pittau and A. D. Polosa, JHEP 0307 (2003) 001.

[9] S. Alioli, P. Nason, C. Oleari and E. Re, JHEP 1006 (2010) 043.

[10] J. R. Andersen and J. M. Smillie, Nucl. Phys. Proc. Suppl. 205-206 (2010) 205; JHEP 1106 (2011) 010.

[11] G. Oderda and G. F. Sterman, Phys. Rev. Lett. 81, 3591 (1998).

[12] J. Forshaw, J. Keates and S. Marzani, JHEP 0907, 023 (2009).

[13] M. Dasgupta and G. P. Salam, Phys. Lett. B 512, 323 (2001).

[14] E. Avsar, Y. Hatta and T. Matsuo, JHEP 0906, 011 (2009).

[15] A. Banfi, G. Corcella and M. Dasgupta, JHEP 0703, 050 (2007).

[16] A. Banfi, G. Marchesini and G. Smye, JHEP 0208, 006 (2002).

[17] Y. Hatta and T. Ueda, Phys. Rev. D 80, 074018 (2009).

[18] R. M. Duran Delgado, J. R. Forshaw, S. Marzani and M. H. Seymour, JHEP 1108, 157 (2011).

[19] M. Deak, F. Hautmann, H. Jung and K. Kutak, arXiv:1112.6386 [hep-ph].

[20] M. Deak, F. Hautmann, H. Jung and K. Kutak, Eur. Phys. J. C 72, 1982 (2012).

[21] S. Alioli, J. R. Andersen, C. Oleari, E. Re and J. M. Smillie, Phys. Rev. D 85, 114034 (2012).

[22] E. Gerwick, T. Plehn, S. Schumann and P. Schichtel, JHEP 1210, 162 (2012).

[23] M. Cacciari, G. P. Salam and G. Soyez, JHEP 0804 (2008) 063.

[24] G. Soyez, Phys. Lett. B 698, 59 (2011).

[25] Z. Nagy, Phys. Rev. Lett. 88 (2002) 122003; Phys. Rev. D 68 (2003) 094002.

[26] M. Cacciari and G. P. Salam, Phys. Lett. B 641 (2006) 57;

M. Cacciari, G. P. Salam and G. Soyez, Eur. Phys. J. C 72 (2012) 1896.

[27] A. D. Martin, R. G. Roberts, W. J. Stirling and R. S. Thorne, Eur. Phys. J. C 28 (2003) 455.

[28] G. Marchesini and A. H. Mueller, Phys. Lett. B 575, 37 (2003).

[29] H. -L. Lai, M. Guzzi, J. Huston, Z. Li, P. M. Nadolsky, J. Pumplin and C. -P. Yuan, Phys. Rev. D 82 (2010) 074024.

[30] C. Marquet, C. Royon, M. Trzebinski and R. Zlebcik, arXiv:1212.2059 [hep-ph].

[31] G. Marchesini and B. R. Webber, Nucl. Phys. B 310, 461 (1988). 